\title{
Three Pricing Models in Modern Portfolio
}

\author{
Wozhi Liu \\ School of Northeastern University, Boston 02115, USA \\ liuwozhi@163.com
}

Keywords: efficient price, portfolio pricing models, Markowitz Pricing Theory, Capital Asset Pricing Model, Arbitrage Pricing Theory.

\begin{abstract}
Before investing, the paramount concern for investors is to have an idea about the expected return from the investment and the risks they are going to take. Determining the efficient price of portfolios by utilizing workable pricing models is the key thing to do for investors. In the history of portfolio pricing, Markowitz Portfolio Theory, Capital Asset Pricing Model (CAPM), and Arbitrage Pricing Theory are three significant pricing methods, which work well under their given circumstances. In this article, I will introduce the three pricing models in modern portfolio, give the mathematical formulas of the three models, and explain them in an understandable way. Furthermore, I will explain the limitations of each model and their connections. Also, I will give my perspective of portfolio pricing by the end.
\end{abstract}

\section{Introduction}

For investors in the stock and security market, one of the most crucial criterions is pricing the financial products efficiently. In other words, assuming the market is efficient, investors are supposed to figure out the efficient price of their purchasing products, including one single stocks, securities, portfolios, and so on, to gain financial benefits. In addition to the supply meets the demand, stock pricing model is to propose the correlations between the price of stocks and factors of financial market indicators. Rational investors will deliberate the interaction between the expected return from their purchasing and various risks related to the financial products. A good pricing model is dramatically valuable for investors to predict and evaluate the proper financial achievement and the risks need to burden before making the final decision; thereafter, the most lucrative investment portfolio is created.

The prototype of modern portfolio theory is Markowitz Portfolio theory, and Markowitz is called the father of the area. His 1952 article on portfolio selection proposed expected return, E, and variance of return, $\mathrm{V}$, of the portfolio as a whole as criteria for portfolio selection, both as a possible hypothesis about actual behaviour and as a maxim for how investors ought to act. Markowitz brought "portfolio selection” to an unprecedented level. Nevertheless, Markowitz's portfolio theory is somehow over idealization, and not suitable in all circumstances. Therefore, other theories came out thereafter, like the Capital Asset Pricing Model (CAPM), which is mainly based on Markowitz portfolio theory, but gets more real financial factors involved. Though Capital Asset Pricing Model (CAPM) is more applicative in the real stock market than Markowitz, it cannot get rid of limitation. To make the pricing theory more reasonable, Arbitrage Pricing Model developed upon Capital Asset Pricing Model was proposed in 1976, and it pushed the study of portfolio pricing to a new stage. These three portfolio theories, Markowitz Portfolio Theory, Capital Asset Pricing Model (CAPM), Arbitrage Pricing Theory, are regarded as the three-classical modern portfolio pricing theories.

The rest of the article structure arrangement is as follow: the second section reviews the fundamental idea of Portfolio Theory, and explain and analysis these tree-classical modern portfolio pricing theories in detail. The third section will introduce the limitations of each theory, and explain the interactions among the tree theories. The last section concludes the entire article, and will mention some other theories declared recently. Furthermore, I will give a future view of portfolio 
pricing theory from where I am standing.

\section{Explanations of three models}

\subsection{Fundamental idea of Portfolio Theory}

First and foremost, we are supposed to understand the fundamental idea about portfolio theory. An investment instrument that can be bought and thereafter sold is called an asset, including stock, currency, option, bond or portfolio [1]. To formulate the theory, we suppose $x_{0}$ be the amount of money invested at time 0 , and $Z_{T}$ be the amount of money received at the selling time $\mathrm{T}$, where $\mathrm{T}$ is the fixed time when the purchased asset is sold. Excluding neither tax nor transaction costs present, the amount of money you receive when selling the asset at time $\mathrm{T}$ is defined as $R=\frac{x_{T}}{\mathrm{v}}$, where $\mathrm{R}$ stands for total return. And the rate of return, $\mathrm{r}$, is defined as $r=\frac{n_{T}-\omega_{w}}{\mathrm{vm}}$. So, $\mathrm{R}=1+\mathrm{r}$, and $\mathrm{r}$ is supposed to be greater than zero to make a profit.

What explained above is the simplest math model suitable for one asset without tax or transaction costs. Now supposed there are $\mathrm{n}$ different assets, and the total return $R_{\mathrm{i}}, \mathrm{i}=1,2 \ldots \mathrm{n}$, is accumulated by the return of each asset. These assets form a portfolio. To formulate the model, we suppose $X_{0 i}$ as the amount of money we invest in asset $\mathrm{i}$, such that $\sum_{i=1}^{\mathrm{n}} X_{0 \mathrm{i}}=X_{0}$, and we restrict $x_{0 i} \geq 0, i=1,2 \ldots n$. Furthermore, let $w_{i}$ be the weights of each asset in the portfolio, such that $X_{0 \mathrm{i}}=w_{\mathrm{i}} X_{0}$. Based on these, the total return $\mathrm{R}$ of the portfolio is defined as

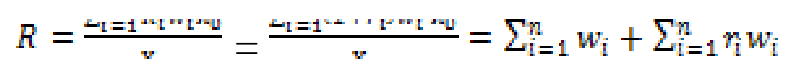

As mentioned above that the sum of $w_{i}$ is one and $\mathrm{R}=1+\mathrm{r}$, hence, we get $r=\sum_{\mathrm{i}=1}^{n} w_{\mathrm{i}} r_{\mathrm{i}}$.

The return of each asset, $r$, is a random variable, because the asset's selling price is unknown when you buy it. Suppose we know the mean for each $n$ asset, we call it $\overline{r_{i}}$, where $i=1,2 \ldots n$. Also, we assign $\sigma_{i}^{2}$ as the variance of asset $\mathrm{i}$, and $\sigma_{i \tilde{i}}$ as the covariance between asset $\mathrm{i}$ and $\mathrm{j}$. Based on these, the expected return of a portfolio ${ }^{\bar{r}}$ is defined as

$$
\left.E[r]=\bar{r}=\rangle, w_{i} E\left[r_{i}\right]=\right\rangle, w_{i} \bar{r}_{i}
$$

The variance $\sigma^{2}$ of the portfolio return is

$$
\begin{aligned}
& \sigma^{2}=E\left[(r-\bar{r})^{2}\right]=E\left[\left(\sum_{i=1}^{n} w_{i} r_{i}-\sum_{i=1}^{n} w_{i} \bar{r}_{i}\right)^{\circ}\right] \\
& =E\left[\left(\sum_{i=1}^{n} w_{i}\left(r_{i}-\bar{r}_{i}\right)\right)\left(\sum_{i=1}^{n} w_{i}\left(r_{i}-\bar{r}_{i}\right)\right)\right] \\
& =E\left[\sum_{i, i=1}^{n} w_{i} w_{i}\left(r_{i}-\bar{r}_{i}\right)\left(r_{i}-\bar{r}_{i}\right)\right] \\
& =\sum_{i, j=1}^{n} w_{i} w_{i} \sigma_{i j}
\end{aligned}
$$

Above is the fundamental idea of Portfolio Theory. [2]

\subsection{Markowitz Portfolio Theory}

Markowitz portfolio theory was proposed by Harry Markowitz who published the article Portfolio selection in Journal of Finance 1952 [1]. Due to his contributions to portfolio theory, Markowitz was conferred Nobel Memorial Prize in Economic Science. The fundamental problem of a portfolio can be formulated in 2 ways, either the investor wants to minimize the variance with respect to a fixed expected return $\bar{r}$ or maximize the expected return given a fixed variance. As mentioned above, $\bar{r}=\sum_{i=1}^{n} w_{i} r_{i}, \sigma^{2}=\sum_{i, j=1}^{n} w_{i} w_{i} \sigma_{i \bar{j}}$, where $\bar{r}$ stands for return, and $\sigma^{2}$ stands for risk. Mathematically, the problem can be formulated as

-Minimize

$$
\sum_{i, j=1}^{n} w_{i} w_{j} \sigma_{i j} \text { subject to } \sum_{i=1}^{n} w_{i} \bar{r}_{i}=\bar{r}, \sum_{i=1}^{n} w_{i}=1
$$

-Maximize

$$
\sum_{i=1}^{n} w_{i} \bar{r}_{i} \text { subject to } \sum_{i, j=1}^{n} w_{i} w_{j} \sigma_{i j}=\sigma^{2}, \sum_{i=1}^{n} w_{i}=1
$$

The above formulas and explanation is known as the Markowitz model [1]. For risk averse, 
formulas (1) is more suitable; whereas formulas (2) is more lucrative for risk lovers. Here, we will mainly elaborate formulas (1), and the second one is quite like formulas (1). Furthermore, we will introduce Lagrange multipliers to explain the main idea precisely. As known that Lagrange multipliers is utilized to find the extremum of a multivariate function $\mathrm{f}$ subject to the constraint $\mathrm{g}=$ 0 , thus it is useful in figuring out the extremum conditions of Markowitz model.

We know that for Lagrange multipliers,

$\nabla f=-\lambda \nabla g$, which means $f^{\prime}{ }_{x}=-\lambda g^{\prime}$. Thus, $f^{\prime}{ }_{x}+\lambda g^{\prime}{ }_{x}=0$. If there are several constraints, the equations will be like $\nabla f+\lambda \nabla g_{1}+\mu \nabla g_{2}=0$

Applying the Lagrange multipliers to the Markowitz model, we will get

$$
\begin{gathered}
\left.f\left(w_{1}, \ldots w_{n}\right)=\right\rangle . w_{i} w_{i j} \sigma_{i \bar{i}} \\
\left.\lambda g_{1}\left(w_{1}, \ldots w_{n}\right)=\lambda(\rangle . w_{i} \bar{r}_{i j}-\bar{r}\right) \\
\left.\mu g_{2}\left(w_{1}, \ldots w_{n}\right)=\mu(\rangle . w_{i}-1\right)
\end{gathered}
$$

Summing up the right side, we get the Lagrangian

$$
\left.L\left(w_{1}, \ldots w_{n}\right)=\text { ᄀ. } w_{i} w_{i} \sigma_{i \bar{p}}+\lambda\left|>w_{i} \bar{r}_{i}-\bar{r}\right|+\mu(\rangle, w_{i}-1\right)
$$

Differentiating $\mathrm{L}$ with respect to $\mathrm{w}$, we will get the linear equation as shown below, and the solution is clearly determined by using linear algebra.

As shown in figure 1, The red dots represent a possible portfolio within the blue line. The efficient frontier consists all possible portfolios, both minimal variance with fixed expected return and maximized expected return with fixed variance.

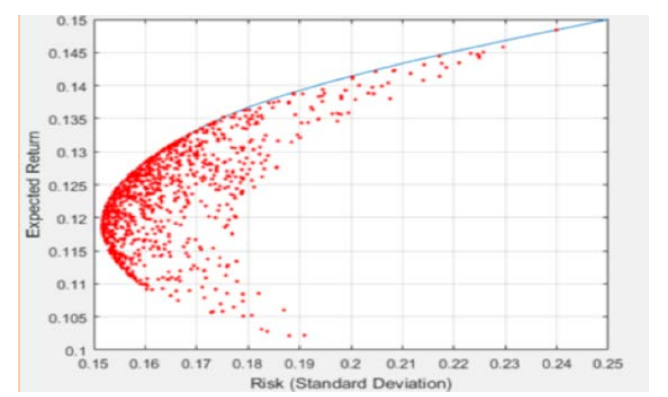

Figure 1 Mean-Variance Efficient Frontier and Random Portfolios.

\subsection{CAPM}

The CAPM builds on the model of portfolio choice developed by Harry Markowitz (1959), and it turns Markowitz's algebraic statement into a testable prediction about the relation between risk and expected return by identifying a portfolio that must be efficient if asset prices are to clear the market of all assets [3]. In 1964 and 1965, Sharpe and Lintner supplement two significant assumptions to CAPM, complete agreement and borrowing and lending at a risk-free rate. the former one claims all investors hold the same perspective and prediction on economic situation, including expected return and potential risks; the latter one states that investors can lent or borrow any amount of money without risk. 


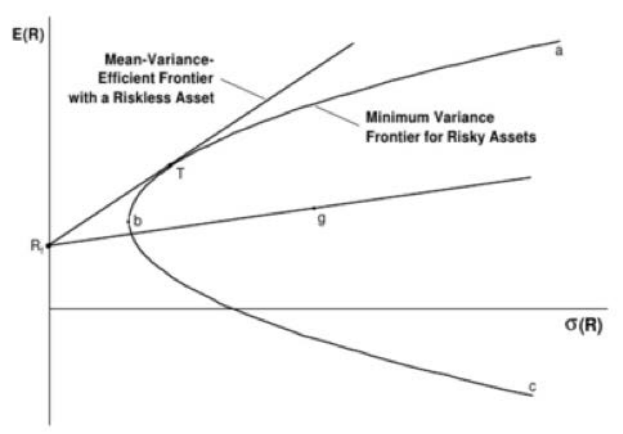

Figure 2 minimum variance frontier, it shows the combinations of expected return and risks of risky assets.

Figure 2 is the minimum variance frontier, which values the combinations of expected return and risk for portfolios of risky assets. The horizontal axis stands for portfolio risks, measured by standard deviation $\sigma$; the vertical axis shows expected return. It is clearly shown that investors, who prefer higher return, must undertake higher risk; and risk adverse must accept lower financial benefits. The curve abc is understandable without risk-free borrowing or lending, in which case only portfolio above b along abc are mean-variance-efficient. However, we add an additional assumption that "borrowing and lending at a risk-free rate" exists; thus, the efficient set becomes a straight line, where $R_{f}$ is the risk-free rate of interest. In terms of this, we can easily understand that any portfolio consists risk-free lending and borrowing and risky portfolio g, which goes along the line from $R_{f}$ through $\mathrm{g}$. If investors plunge a proportion $\mathrm{x}$ of funds in risk-free security and $1-\mathrm{x}$ in risky portfolio g, the formula of expected return and standard deviation of return of risk-free security and risky g will be like the following

$$
\begin{gathered}
R_{p}=x R_{f}+(1-x) R_{q} \\
E\left(R_{p}\right)=x R_{f}+(1-x) E\left(R_{g}\right) \\
\sigma\left(R_{p}\right)=(1-x) \sigma\left(R_{q\}^{\prime}} x \leq 1\right.
\end{gathered}
$$

To determine the relations between expected return and risks, that means to price risky assets, Capital Market Line, CML is not eloquent enough, thus we need to supplement Security Market Line, SML. And the formula is

$$
r=r_{0}+\beta_{M}\left(r_{M}-r_{0}\right)
$$

which is the precise expression of CAPM model. It is obvious that expected return of a risky asset consists two parts, return of non-risk asset and Market risk compensation. Excluding unsystematic risk, which could be eliminated easily, we only consider the impact of systematic risk. Suppose we are trying to price a single asset $i$, the expected return of i could be formulated as

$$
r_{\mathrm{i}}=r_{0}+\beta_{\mathrm{Mi}}\left(r_{\mathrm{m}}-r_{0}\right)
$$

where $r_{0}$ is return of non-risk asset, $\beta_{\text {Mi }}$ stands for the relation of risk between asset $\mathrm{i}$ and standard portfolio $\mathrm{M}$, and it is calculated as $\beta_{\mathrm{Mi}}=\sigma_{\mathrm{Mi}} / \sigma^{2}$. Because of taking risks, investors are supposed to be offered a reward, and the reward is $\left(r_{M}-r_{0}\right)$. Under this circumstance, $\beta_{M i}\left(r_{m}-r_{0}\right)$ measures the risk premium. Up to now, we realize that the index to measure risk is $\beta_{M i}$ instead of the variance of $i$, and we come up with three circumstances for the Market Price of asset $\mathrm{i}$ :

1. $\beta_{M i}$ is greater than 1 , which means asset $\mathrm{i}$ is aggressive, such that its price ascends as the price of $\mathrm{M}$ increases.

2. $\beta_{M i}$ is less than 1 , which means asset $\mathrm{i}$ is defensive, such that its price goes adversely with the price of $\mathrm{M}$.

3. $\beta_{M i}$ equals 1 , which means $\mathrm{i}$ is mutual, such that its price goes simultaneously with the price of $\mathrm{M}$. 


\subsection{Arbitrage Pricing Theory}

Arbitrage Pricing Theory was proclaimed by Stephen Ross 1976, and it is a substitute for the Capital Asset Pricing Model (CAMP) in that both assert a linear relation between assets' expected returns and their covariance with other random variables [4]. Arbitrage means purchasing an asset at a low price and sell it at a high price to gain financial benefits. From mathematical point, we may assume the price vector of an asset is $S=\left[S_{1} ; S_{2} ; \ldots S_{N}\right]$, and payoff matrix $X=|\vdots \quad \vdots \quad \vdots \quad|=X\left[\theta_{1} \ldots \theta_{N}\right]$. The Asset Pricing Model of Arbitrage Pricing Theory is the mapping from $\mathrm{X}$ to $\mathrm{S}$. When considering an asset portfolio $\theta=\left[\theta_{1} \ldots \theta_{N}\right]$, we assume its price at time 0 is $S^{T} \theta$, and payoff vector at time 1 is $\mathrm{X} \theta$, then there are 3 circulations that arbitrage opportunity appears:

- $S^{T} \theta<0$ and $\mathrm{X} \theta=0$

- $S^{T} \theta=0$ and $\mathrm{X} \theta>0$

$\bullet S^{T} \theta<0$ and $\mathrm{X} \theta>0$

We can formulate the Arbitrage Pricing Theory as follow:

$$
r_{i}=r_{F}+>\beta_{i j} \lambda_{i}
$$

where $r_{1}$ stands for the expected return of $\mathrm{i}, r_{F}$ stands for the return of all portfolios having no sensitiveness of factor risk, $\lambda_{i}$ is the factor premium of the $i^{\text {th }}$ factor, and $\beta_{i j}$ stands for the sensitiveness of asset $\mathrm{i}$ confronting all kinds of factor risks. The risky factor could be just one or multiple.

\section{Discussion}

Markowitz Portfolio Theory is the pioneer of analysing the expected return of a venture investment and its uncertain risks, to determine the most lucrative portfolio, and it stands for the new era of modern portfolio theory. The 2 most significant conclusions from Markowitz Portfolio Theory are first unsystematic risks of venture investment could be avoided by diversification, and second the only rational portfolio to be invested is the point of tangency between Mean-variance efficient frontier and random portfolio curve. However, Markowitz did not determine a valid assumption to the interaction of expected return between each asset. To set up the efficient frontier, we have to determine each covariance between any two assets. Though the covariance is precise, the calculation is over sophisticated. What is more, Markowitz's theory focus on personal investors, which means it does not give an efficient reference to the broad market. Sharpe and Linter proposed Capital Asset Pricing Model based on Markowitz Portfolio Theory to solve this problem. They assume that risky factors, which influence the price of assets, are based on the market level. In other words, we only need to know the factors of standard portfolio $\mathrm{M}$ and parameter $\beta$, but we do not have to know every covariance. This process simplifies the complicated calculations of Markowitz Portfolio Theory. Furthermore, CAPM figure out a consequential problem, that if all investors build their portfolios following Markowitz Portfolio Theory, then what factors we may consider pricing the assets, and how to price it. In other words, instead of focusing on personal investor, Sharpe and Linter push their view to the whole market. However, Capital Asset Pricing Model is still based on a series of premises, so it is different from the real market. Thereafter, Stephen Ross proposed Arbitrage Pricing Theory 1976 pushing the asset pricing to a higher stage. Compared to Markowitz and CAPM, Arbitrage Pricing Theory gives us a more prevailed formula based on much less premises; for instance, it does not assume a non-risk lending and borrowing, and it does not require investors making purchasing decisions based on return and covariance. More importantly, Arbitrage Pricing Model could be influenced by kinds of risky factors, but it does not matter what the factors are, because we do not necessary to know it within the formula. Arbitrage Pricing Theory assume investors prefer high return with a constant risk, which is seemingly correct in the real market. 


\section{Conclusion}

Maintenance and appreciation in asset value is what investors care about, and more investors are getting interested in venture investment; this accelerates the innovation of financial industry and becomes the main impetus of the economic development for countries. To gain the expected financial benefits, investors are supposed to utilize scientific pricing models to determine the value of portfolios. Looking back the development history of portfolio pricing, from the precursor Markowitz Portfolio Theory, to Capital Asset Pricing Model (CAPM), then Arbitrage Pricing Theory, and more and more precise pricing model proposed thereafter; the research of asset pricing is developing on a positive way at an accelerating pace.

However, theories are sometimes idealized, that they are only practicable under a series of circumstances. Controversially, the real financial market is never idealized that all circumstances have impacts on the result of portfolio pricing. Thus, to come up with more precise models to apprize portfolios to gain expected financial benefits, we still have a long way to go.

\section{References}

[1]. Zhao, Yingjie. "Research on the Multi-factor model for Stock Pricing”. Xi'an University of Architecture and Technology. 2009

[2]. Amu, Farhad, and M. Millegard. "Markowitz portfolio theory." Cite seer. Retrieved on 12.15 (2009): 1.

[3]. Fama, Eugene F., and Kenneth R. French. "The capital asset pricing model: Theory and evidence." The Journal of Economic Perspectives 18.3 (2004): 25-46.

[4]. Huberman, Gur. Arbitrage pricing theory. No. 216. Staff Report, Federal Reserve Bank of New York, 2005. 